\title{
Photoacoustic Tomography System
}

\author{
J. D. Martínez-Ramírez ${ }^{1}$,R. Quispe-Siccha ${ }^{2}$, C. García-Segundo ${ }^{3}$, \\ F. J. González ${ }^{4}$, R. Espinosa-Luna ${ }^{5}$, G. Gutiérrez-Juárez ${ }^{\star 6}$ \\ 1,4 Instituto de Investigación en Comunicación Óptica, UASLP. \\ Av. Karakorum, 1470 Lomas 4a. 78210 San Luis Potosí, SLP México. \\ ${ }^{2,3}$ Centro de Ciencias Aplicadas y Desarrollo Tecnológico de la UNAM. \\ Circuito Exterior S/N C.P. 04510 Cd. Universitaria, \\ México, D. F. A. P. 70-186 \\ ${ }^{5}$ Centro de Investigaciones en Óptica. \\ Loma del Bosque 115, Lomas del Campestre, \\ C. P.: 37150, León, Gto., México. A. P. 1-948.

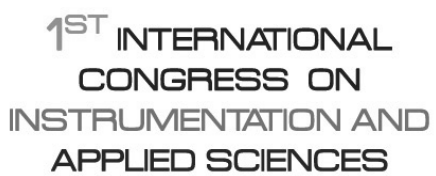

${ }^{6}$ División de Ciencias e Ingenierías,

Universidad de Guanajuato-Campus León.

Loma del Bosque 103, Lomas del Campestre,

C. P.: 37150, León, Gto., México. A. P.: E-143.

*ggutj@fisica.ugto.mx

\section{ABSTRACT}

Based on the pulsed photoacoustic effect, we set up an experimental system to obtain bi-dimensional images of optically-opaque samples embedded within the bulk of turbid medium. The turbid medium was made of agar gel mixed with single-sized nanoparticles; with these materials we induce an optical absorption and an optical scattering like that appearing in human tissues. The PA signals are generated from the absorption processes in the buried target, and then traveling through the bulk of scatter medium. The optical absorption properties and the shape of target, defines the amplitude and shape of the PA signals. This time the laser pulses are set from a pulsed Nd: YAG laser, with pulse width of $10 \mathrm{~ns}$, at rate repetition of $10 \mathrm{~Hz}$ and wavelength set at $1064 \mathrm{~nm}$. The signals generated in this way are registered by means of an ultrasonic transducer with resonance cut at $10 \mathrm{MHz}$. The sample was rotated to obtain as many as 36 projections which are used to feed an image reconstruction forward-projection algorithm based on the Radon Transform. As result we obtain 2D tomographic slices of three different samples.

Keywords: Photoacoustic imaging, radon transform, tomography.

\section{RESUMEN}

Basados en el efecto fotoacústico pulsado, se desarrolló un dispositivo experimental para generar imágenes bidimensionales de muestras ópticamente opacas ocultas en un medio turbio. El medio turbio se hizo de agar y nanopartículas monodispersas, con estos materiales indujimos una absorción y esparcimiento óptico semejante al de los tejidos humanos. La señal PA se genera por la absorción óptica del la muestra; una vez producida viaja a través del medio esparsor. Las propiedades de ópticas de la muestra así como la forma de éste definen la amplitud y forma de la señal PA. En el sistema fotoacústico se utilizaron pulsos láser de Nd: YAG de $10 \mathrm{~ns}$, con frecuencia de repetición de $10 \mathrm{~Hz}$, y longitud de onda de $1064 \mathrm{~nm}$. Las señales generadas fueron registradas por un transductor ultrasónico con frecuencia de corte de $10 \mathrm{MHz}$. Tanto la muestra como el medio esparsor se rotaron para obtener 36 proyecciones, las cuales fueron utilizadas para alimentar un algoritmo de reconstrucción de imágenes basado en la transformada de Radon. Como resultado se obtuvieron imágenes tomográficas 2D de tres muestras distintas.

\section{Introduction}

Because of its non-invasive properties, in last decades, optical tomographies have been accepted as a diagnostic tool in biomedical imaging researches. The basis of the technique is to exploit the light-tissue interaction and its optical contrast in terms of optical absorption, from where one can obtain images. Notice that, since the tissue presents large amounts of light scattering, the option of optically reconstructing a tomographic image is not good enough. However, the photoacoustic tomography (PAT) [1-7] is a novel imaging technique that combines the high optical contrast of the optical tomographies with the high resolution of the ultrasound imaging. The PAT is 
based on the photoacoustic (PA) effect which consists of the generation of ultrasound waves as result of the absorption of electromagnetic energy. All energy beams, as those comprised of visible and infrared radiation, X-rays, electrons, protons, ions or other particles, are capable of generating the PA effect when they interact with matter, whilst in PAT the method of producing PA ultrasound waves is by means of using laser light pulses, whose pulse-width is in the range of nanoseconds. The laser light in these sorts of applications consists of non-ionizing radiation, which is not harmful to the human body [1]. The advantage of PAT over purely optical imaging is primarily one of the signal propagation distance and hence, the size and depth of the region under investigation.

In the biological media, such as the human tissue, the propagation of light is dominated by the optical scattering. The difficulty of modeling the optical scattering in complex media, such as the human tissue, imposes heavy limits to the usefulness of optical imaging. For example, human skin has a scattering coefficient of about $200 \mathrm{~cm}-1$ at red wavelengths, while human aortic tissue has a scattering coefficient of about $300 \mathrm{~cm}-1$ [7, 8]. In both cases, due to the scattering, the light mean free path is of few tens of microns. Notice that the light propagation through distances greater than one or two millimeters results in loss of coherence and of polarization; and hence, cancel the ability of imaging. In contrast the acoustic wave propagation, such as the PA kind, is not affected by the optically turbid media. The turbid media affects the acoustic waves (PA waves) only over its generation, since this is dependent on the optical absorption. Acoustic propagation in tissue can be characterized by the reflections on the interface and by viscoelastic attenuation.

The ultrasound wave that is generated by the pulsed light is the initial source of pressure. This burst travels from its initial position toward the surface, where is detected at the positions where the sensor is placed on the tissue. Finally, the recorded time resolved signals are used to reconstruct the initial pressure distribution. Usually, detectors based on piezoelectric transducers are used to record the acoustic waves due to their flexibility to be manufactured in arbitrary shapes, their high sensitivity, and their capability to perform in parallel detection. Piezoelectric parallel detection has been widely used [2-7] to make PA imaging.

In this work we use a single commercial piezoelectric detector to obtain two dimensional images by means of processing its output using algorithms similar to those used in X-ray tomography, based on the Radon transforms. The programming code was implemented in LABVIEW environment. The dummy samples are made of agar mixed with $\mathrm{SiO}_{2}$ nanoparticles and had three different shapes. These samples were embedded in phantoms made also of agar, mixed with $\mathrm{TiO}_{2}$ nanoparticles. In this way we built up samples that possess the optical and mechanical properties of background soft-scatter tissue and a cancerous lesion, in close resemblance to the human tissue.

\section{Materials and methods}

\subsection{Sample preparation}

All imaging experiments were performed on dummy models, the phantoms. The phantom materials were selected to qualitatively and quantitatively simulate the optical and mechanical properties of background soft-scatter tissue and a cancerous lesion $[7,8]$. Both the placement and the size of the lesion were chosen to closely mimic clinical environment. Overall, each phantom was a $60-\mathrm{mm}$ (axial) by $40-\mathrm{mm}$ (radial) cylindrical-shaped object. To test the sensitivity and resolution of our system, we used different types of nanoparticles mixed in agar to represent lesions. Small pieces of these phantoms were embedded in three cylindrical phantoms of the kind described above. In each case for the placing of the lesion, special care was taken to set it up along the central vertical line of the cylinder. The schematic representation and a picture of the lesion phantoms are shown in Figure 1. The lesion phantoms, with nanoparticles inclusion, were prepared as follows: $20 \mathrm{ml}$ of distilled water are heated up to their boiling point, and then agar (Bioxon, Cat 211900) and $\mathrm{SiO}_{2}$ nanoparticles are added under smooth and continuous stirring. The cylindrical phantom is prepared in similar way: we get boiled distilled water, then Agar powder and this time $\mathrm{TiO}_{2}$ nanoparticles are added to this water, keeping continuous and smooth stirring of the mix until a homogeneous mix is obtained. The nanoparticle concentration used in the preparation of lesion 
(a)

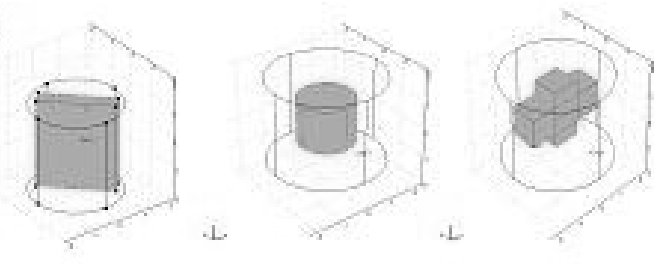

(b)

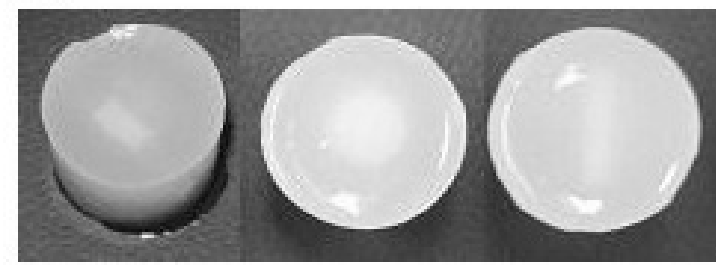

Figure 1. The schematic-cartoon representation of the phantom samples is exhibit in (a), in (b) is exhibit an actual picture of the lesion-phantoms.

produces an optical absorption coefficient of 1.81 $\mathrm{cm}^{-1}$. The optical absorption and the scattering coefficients of the background soft-scatter phantom were $1.93 \mathrm{~cm}^{-1}$ and $8.02 \mathrm{~cm}^{-1}$, respectively. These coefficients were obtained by using an integrating sphere-arrangement, an illuminating wavelength of $1064 \mathrm{~nm}$ and a photodetector, according to the method described in chapter 2 of Ref. 8. Both $\mathrm{SiO}_{2}$ and $\mathrm{TiO}_{2}$ nanoparticles, are synthesized in CCADET-UNAM laboratories, had an average diameter of $600 \mathrm{~nm}$ with size distribution no larger than the $20 \%$ of the mean diameter.

\subsection{Experimental setup}

The experimental setup as used in our experiments, is based on a mechanical scanning mechanism, and is schematically shown in Figure 2. In this system, we used a Q-switch Nd: YAG laser (Brilliant, Quantel) operating at $1064 \mathrm{~nm}$ wavelength, with pulse duration of $10 \mathrm{~ns}$ and a repetition rate of $10 \mathrm{~Hz}$, these laser pulses are directly pumped on the phantom. The fluence of infrared radiation was $90 \mathrm{~mJ} / \mathrm{cm} 2$; this is well within the safety standard for a Nd: YAG laser at 1064 nm. A $3.5 \mathrm{MHz}$ transducer (piezo-composite, 1.5 $\mathrm{cm}$ diameter, V383 PANAMETRICS) is used to register the acoustic signals. The transucer is
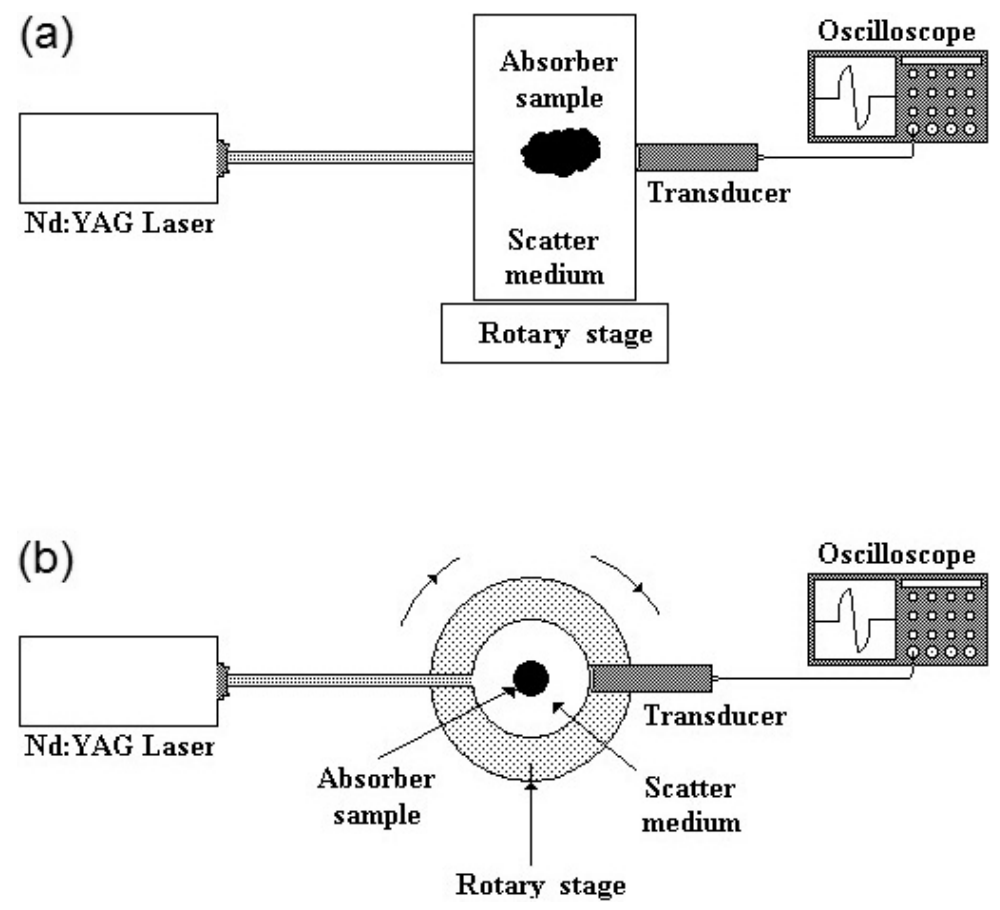

Figure 2. The schematic experimental setup, (a) side view, and (b) top view. 
firmly attached to the phantom via ultrasound gel, in order to get a better acoustic coupling. The receiver phantom is set on top of a rotary stage, whose rotation axis is set to be perpendicular to the laser beam incidence. One set of data is taken at 36 positions when the phantom is scanned circularly over $360^{\circ}$. The PA signal, without amplification, fed to a Digital Phosphor Oscilloscope (TDS5104B, Tektronix), which sends automatically the PA signal to personal computer.

\section{Results and conclusions}

For each position of the phantom a PA signal was obtained. In Figure 3, a typical PA signal as a function of time is shown. Data set fed a LABVIEW program based on the Radon transform [9]. Figure 4(a) gives the image of the cylindrical-shape lesion phantom, Figure 4(b) presents the image of the cross-shaped lesion phantom, and finally Figure 4(c) shows the image of the planar lesion phantom. From the reconstructed images we found that these are in good agreement with the actual objects. Some multiple-reflection patterns are noted in the background, particularly at the interface between the soft-scatter phantom and the lesion solid phantom. Closely examining these images, we observe that the multiple-reflection patterns exhibit an oscillating pattern that is similar to the acoustic pressure wave distribution (see Figure 3). This result may be improved by enlarging the receiving frequency range. Such action would help to average out the frequency associated artifacts and to increase the number of data sets. So far the tomographic images have been achieved using experimental models of optical high contrast of

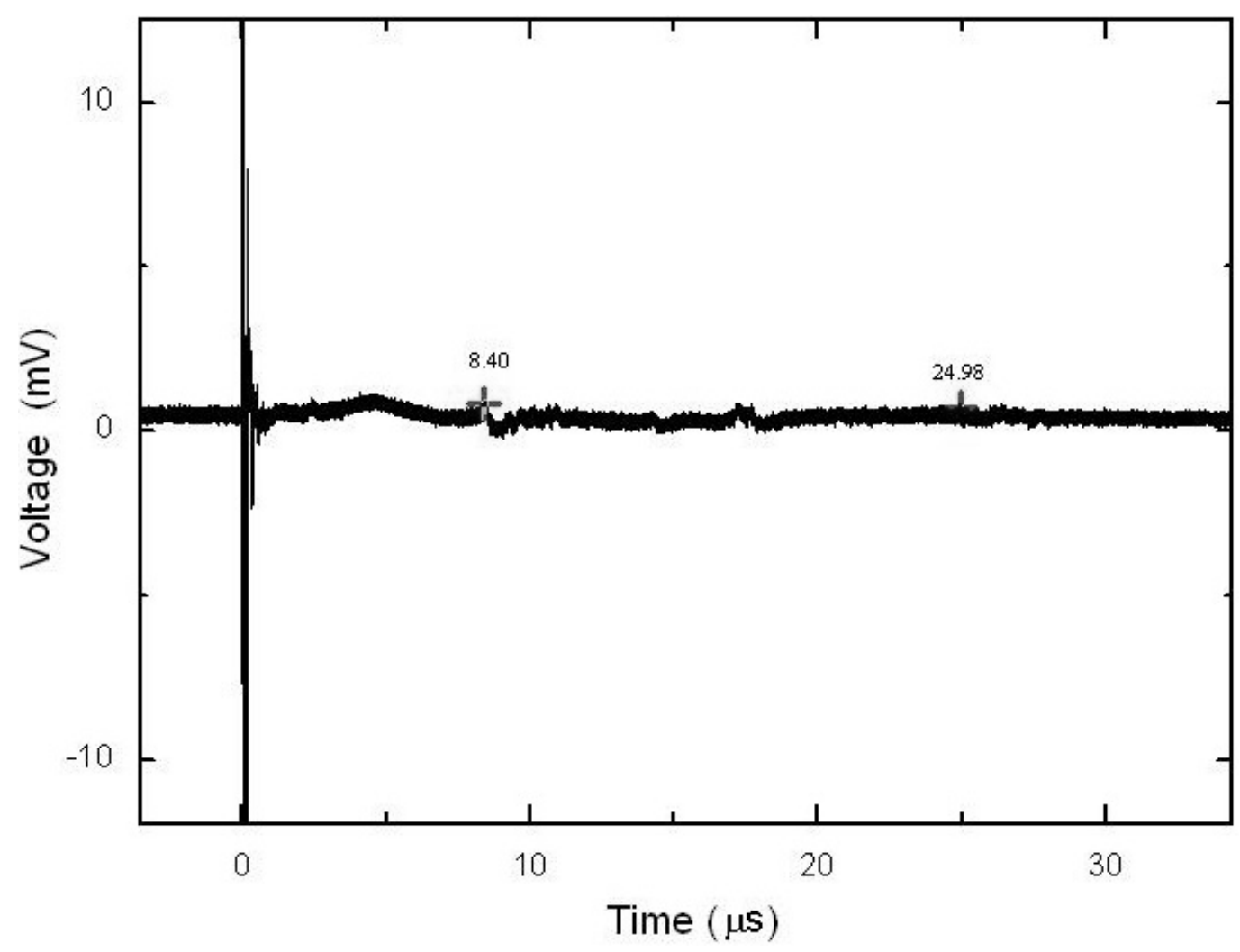

Figure 3. Characteristic PA signal as registered for each position on the phantom. 

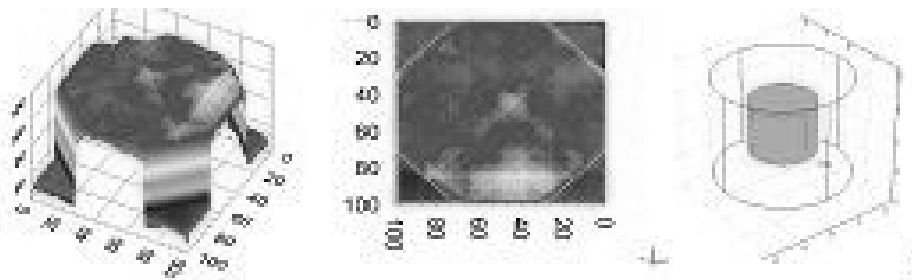

(a)
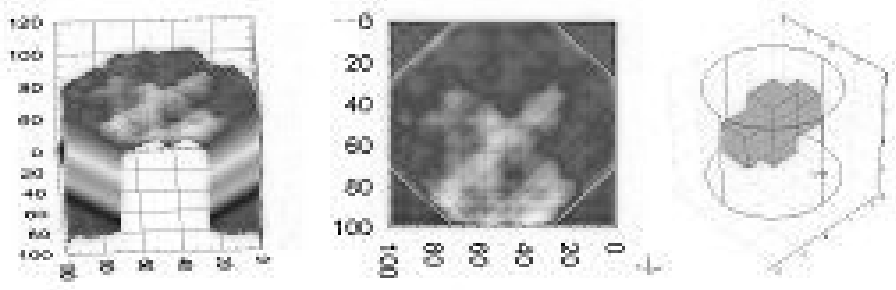

(b)
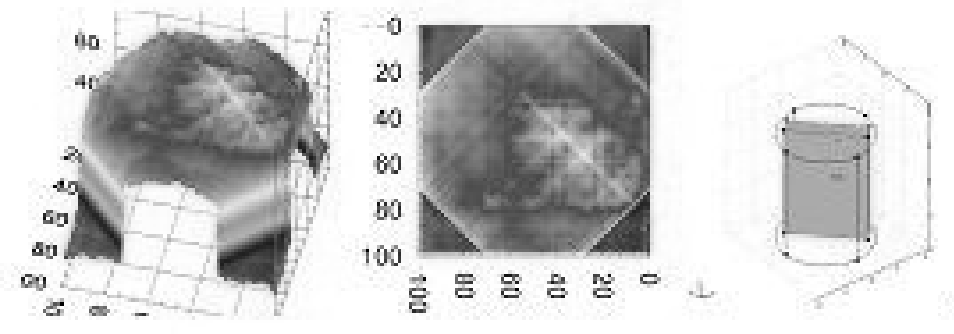

(c)

Figure 4. 2D tomographic slice for (a) cylindrical, (b) cross, and (c) planar shape.

large dimensions of fixed optical and mechanical properties $[10,1]$. In this work we present results for low-optical-contrast models, based on colloidallike discrete structures; therefore, these are optically and mechanically diffusive. These are conditions closer to the actual real biological models. Furthermore, among other advantages of this experimental model is that we can manipulate in controlled way the mechanical and optical properties. In relation to the actual images, we have established threshold conditions on the optical contrast that defines the limits of applicability of this range of methodologies. To sum up, we have demonstrated a reconstruction process based on the Radon transform that allows us to obtain semiquantitative photoacoustic images of small nanoparticle-containing objects.

\section{References}

[1] Minghua X. and Lihong V. W., Photoacoustic Imaging in biomedicine. Rev. Sci. Intrum. Vol. 77, No. 4, April, 2006, pp. 041101-041122.

[2] Ermilov S. A., Khamapirad T., Conjusteau A., Leonard M. H., Lacewell R., Mehta K., Miller T., and Oraevsky A. A., Laser Optoacoustic Imaging System for Detection of Breast Cancer, J. Biomed. Opt. Vol. 14, No. 2, March/April, 2009, pp. 024007-024012.

[3] Ashkenazi S., Hou Y., Huang S., Buma T., and O'Donnell M., High Frequency Optoacoustic Transducers for Ultrasonic and Photoacoustic Imaging (CRC Press, 2009)

[4] Burgholzer P., Hofer C., Paltauf G., Haltmeier M., and Scherzer O., Thermoacoustic tomography with integrating area and line detectors, IEEE Trans. Ultrason. 
Ferroelectr. Freq. Control. Vol. 52, No. 9, September 2005, pp. 1577-1583.

[5] Haltmeier M., Scherzer O., Burgholzer P., Nuster R., and Paltauf G., Thermoacoustic tomography and the circular Radon transform: Exact inversion formula, Math. Models Meth. Appl. Sci. Vol. 17, No. 4, April, 2007 pp. 635-655.

[6] Paltauf G., Nuster R., Haltmeier M., and Burgholzer $P$., Experimental evaluation of reconstruction algorithms for limited view photoacoustic tomography with line detectors, Inverse Probl. Vol. 23 No. 6, December, 2007, pp S81-S94.

[7] Duck FA (1990) Physical Properties of Tissue: a Comprehensive Reference Book. Academic Press Inc., San Diego

[8] Markolf H. N., Laser-Tissue Interactions Fundamentals and Applications, Third, Enlarged Edition, Springer-Verlag, 2007, Berlin, Heidelberg.

[9] The Radon Transform by Carsten Høilund, Aalborg. Univeristy, VGIS, 07gr721, Nov 2007.

[10] Wenfeng Xia, Daniele Piras, Michelle Heijblom, Wiendelt Steenbergen, Ton G.van Leeuwen, and Srirang Manohar, Poly(vinyl alcohol) gels as photoacoustic breast phantoms J. Biomed. Opt. Vol 16, No 7, Jul, 2011, pp-075002

\section{Acknowledgments}

This work is supported by CONACyT-Mexico grant 83945 and by Instituto de Ciencia y Tecnología del Distrito Federal, Mexico, through a grant contracted with CCADET-UNAM. 